\title{
Paraplegia
}

\section{Proximal Femurectomy for Decubitus Ulceration in the Spinal Cord Injury Patient}

\section{J. W. Peters, MD, G. E. Johnson, MD, FRCS(C)}

Department of Orthopaedic Surgery, Health Sciences Centre and University of Manitoba, Rm GF311, 820 Sherbrook Street, Winnipeg, Manitoba, Canada R3A $1 R 9$.

\section{Summary}

The results of proximal femoral resections in nine patients were analyzed after an average four year follow-up, together with a retrospective analysis of the patients. The indication for surgery was decubitus ulceration that had failed to respond to multiple previous treatments. The decubitus ulcers were associated with septic arthritis, osteomyelitis, hip dislocation and ankylosis. All patients achieved short term healing with $50 \%$ achieving long term healing. The incidence of subsequent surgery for decubitus ulcers decreased significantly. No patient suffered a deterioration in function.

Key words: Decubitus ulceration; Paraplegia; Quadriplegia; Femoral resection.

Decubitus ulceration continues to be a costly and serious problem in spinal cord injury patients varying from 10 to $50 \%$, and with a cost estimate of $\$ 15000$ per ulcer treated (Richards and Meyer 1981). Abnormal pressure and shear forces have been implicated (Bennet et al., 1984), but the aetiology of decubitus ulcers remains unclear (Patterson and Fisher 1986). Prevention of decubitus ulcers by exercises and special devices for seating have been recommended and are widely used (Ferguson-Pell et al., 1980) (Perkash et al., 1984).

Despite good preventive care decubitus ulceration sometimes occurs. Operative intervention, when necessary, must focus on the restoration of a supple cushion of well nourished soft tissue (Reichart, 1986) without underlying bony prominences. Patients with severe decubitus ulcers may require one or several operative procedures. Failure to achieve healing after multiple operations can result in the situation where no further tissue is available for reconstruction, then, hip disarticulation may be deemed necessary, but we feel that a less radical and disfiguring operation should be considered, consisting of excision of the 
Table I Level of neurological injury

\begin{tabular}{cc}
\hline Number & Neurological level \\
\hline 1 & C6 \\
3 & C7 \\
1 & T1 \\
1 & T8 \\
1 & T12 \\
2 & L1 \\
\hline
\end{tabular}

proximal portion of the femur which preserves the limb while providing tissue for closure of the decubitus ulcers.

Proximal femoral resections in spinal cord injury patients is not a new procedure, thus Eltorai (1983) reported on femoral head and neck excisions, and Stewart and Comarr (1971) and O'Hare (1964) on subtrochanteric resections. Each of these were carried out primarily for decubitus ulceration. Ducharme (1967) described the removal of the proximal half of the femur in patients with decubitus ulcers, spasms and contractures. The purpose of this paper is to report our experience with proximal femoral resections, including a more detailed follow-up evaluation concerning the benefits.

\section{Method}

Examination of our hospital's medical records revealed that 9 proximal femoral resections were performed in spinal cord injury patients from 1976 to 1986. These 9 charts were reviewed retrospectively to determine demographic data, the indications for and the details of the surgical technique, as well as the complications and the results.

Follow-up studies were arranged in co-operation with the Spinal Cord Injury Unit at our hospital. Four patients were available for interview and examination by the authors. Three patients lived in geographically isolated areas; 1 was interviewed by telephone while 2 did not have a telephone and were contacted through their family physician. The 2 remaining patients were known to be deceased; in neither case was death attributable to surgery. Follow-up was available on 1 of the deceased patients via his hospital charts while the other deceased patient was lost to follow-up.

\section{Results}

All 9 patients were males with a mean age of 21 years at the time of spinal injury, and 32 years when undergoing proximal femurectomy. Eight cases were traumatic in aetiology while 1 patient had a meningomyelocele. The neurological level of injury is indicated in Table I.

The indication for surgery in 8 patients was recurrent decubitus ulcers refractory to previous surgical management. Associated with the decubitus ulcers were osteomyelitis in 6 cases, hip dislocation in 5 cases and septic arthritis in 4 of the cases. In one patient, ankylosis of the hip was the primary indication for surgery.

These 9 patients had undergone a total of 25 procedures for decubitus 
Table II Surgical procedures undertaken for decubitus ulceration prior to proximal femoral resection

\begin{tabular}{lc}
\hline \multicolumn{1}{c}{ Procedure } & Number \\
\hline Excision and split & 8 \\
thickness skin graft & 11 \\
Myocutaneous tissue flap & 3 \\
Ischiectomy & 3 \\
\hline
\end{tabular}

Table III Early complications of proximal femoral resection and their management

\begin{tabular}{lcl}
\hline \multicolumn{1}{c}{ Complication } & Number & \multicolumn{1}{c}{ Management } \\
\hline Skin edge necrosis & 2 & Local care \\
Hematoma & 1 & Drainage \\
Osteomyelitis & 1 & Revision of femoral resection \\
\hline
\end{tabular}

Table IV Late complications of proximal femoral resection and their management

\begin{tabular}{lcl}
\hline \multicolumn{1}{c}{ Complication } & Number & \multicolumn{1}{c}{ Management } \\
\hline Myositis ossificans & 1 & None required \\
Decubitus ulceration & 4 & Local care \\
$\quad$ Ischeal ulceration (2) & & Local care \\
Lateral thigh ulceration & & Ischiectomy \\
Perianal ulceration & & \\
\hline
\end{tabular}

ulceration prior to the time of proximal femoral resection (Table II). All resections were carried out via a lateral approach, following previous flap margins and incision lines. Sinus tracts and ulcers were incorporated in the incision. The femur was exposed by subperiosteal dissection and osteotomised at least 2 $\mathrm{cm}$ below the lesser trochanter. After removal of the proximal segment the acetabulum was carefully debrided. Four wounds were closed over drains while 5 were packed open with loose approximation of tissues. In 1 patient with severe ulceration and knee flexion contracture more than half of the femur was resected. Peri-operative antibiotic coverage was used against the predominant organisms isolated on culture.

Follow-up was obtained on 8 patients with a mean post-operative period of 4 years. The 1 patient with no follow-up was known to have died 3 years postoperatively and had been a poor attender at outpatient clinics. Early complications (Table III) were limited to the wound and did not result in significant morbidity. At the time of discharge 8 of 9 decubitus ulcers were healed and the other ulcer healed subsequently after discharge home. Late complications (Table IV) consisted of recurrent decubitus ulceration, and myositis ossificans.

Functional status was evaluated subjectively in 5 patients based on cosmesis, sitting and transferring. Only 1 patient felt that the cosmesis of the limb was a problem. Sitting and transferring were improved in 2 patients and unchanged in 3. All patients were sitting in a wheelchair at least 16 hours a day and transferring independently, except the patient with a C6 neuro- 


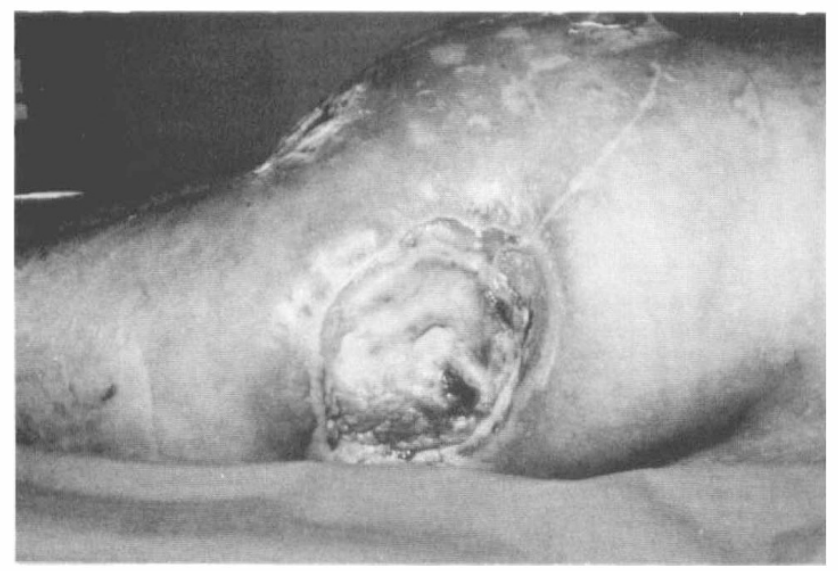

Figure 1 Large decubitus ulcer over greater trochanter despite several previous operative attempts at treatment.

logical level lesion. No patient suffered functional deterioration as a result of the proximal femoral resection.

\section{Discussion}

Our patients consisted of a group of young men, most of whom had traumatic spinal cord injury. Our demographic data correlate well with that of Stewart and Comarr (1971) in their review of a 25-year experience. Our patients had a slightly longer period of time, 11 years on average, from time of cord injury to the time of femoral resection. The level of neurological injury was variable but all patients were confined to a wheelchair and had insensitive lower extremities. In the 11-year interval from injury to proximal femoral resection these patients underwent numerous operative and non-operative treatments for decubitus ulceration. A record of all operative procedures was kept which indicates that a total of 25 surgical procedures were done in these 9 patients over the period prior to femoral resection for decubitus ulcers

Decubitus ulceration (Fig. 1) was the major indication for proximal femoral resection. Because these patients had undergone previous multiple operations further less radical surgical procedures were, in consultation with the Department of Plastic Surgery, considered to be inadequate. The conditions associated with decubitus ulcers in spinal cord injury patients have been shown to increase the difficulty of treatment. Freehafer (1963) described the association of septic arthritis, hip dislocation, osteomyelitis and decubitus ulceration (Fig. 2). One hip dislocation was non-septic (Baird et al., 1986) but there were associated decubitus ulcers, and therefore proximal femoral resection was chosen. The alternative procedure of hip disarticulation, is more radical and disfiguring and is not readily accepted by patients. The 1 patient undergoing proximal femoral resection for ankylosis had previously undergone a femoral head excision resulting in ulcer healing, but complicated by myositis ossificans and hip ankylosis in a non-functional position. Overall our indications for surgery are similar to those reported in other series. 


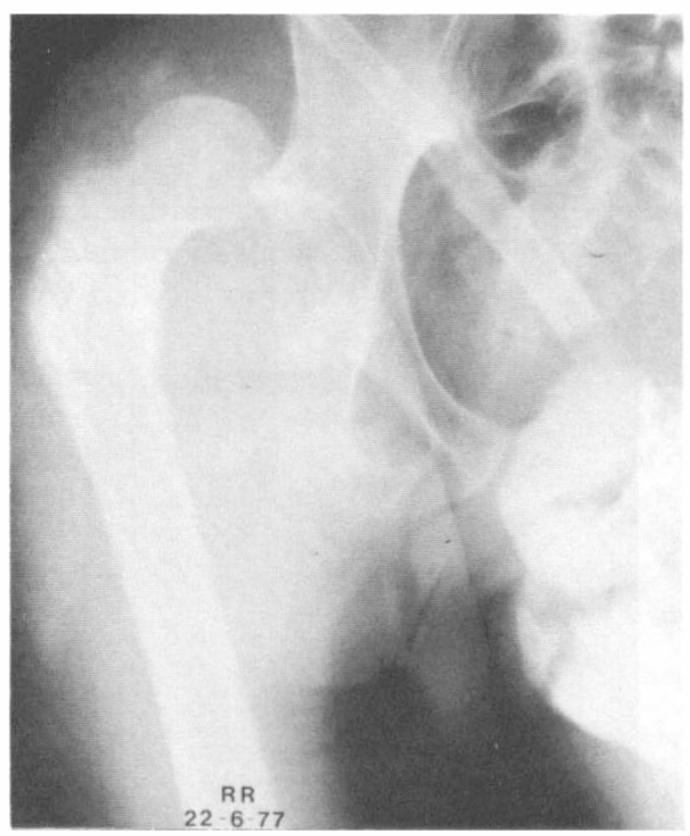

Figure 2 Radiograph of patient with trochanteric decubitus ulcer associated with septic hip dislocation, osteomyelitis and previous resection of greater trochanter.

The surgical approach is similar to that described by Ducharme (1967). We would like to emphasise that an adequate length of femoral shaft must be resected to prevent ankylosis. We have found that a resection about $2 \mathrm{~cm}$ below the lesser trochanter is sufficient as opposed to the midshaft level annotated by Ducharme (Fig. 3). Complete excision of the abscess cavity and granulation tissue in the acetabulum is an important consideration in technique to ensure eradication of infection. Primary closure was chosen in only carefully selected cases; when any question of contamination existed, secondary closure was selected. The incidence of wound complications was not related to choice of closure. The local wound complications experienced are not unexpected considering the quality of the tissue operated on, most responded to local care. The one case of osteomyelitis was a reflection of inadequate excision of the infected femur and resulted in prolonged wound drainage. In 4 cases all decubitus ulcers were not excised at the time of femoral resection. These patients subsequently had excision of the ulcers and myocutaneous transposition flap using redundant tissue available after the femoral resection.

All patients were healed a short time after discharge from hospital. Four patients have remained healed with no recurrence of their decubitus ulcer for a mean group follow-up of 5 years (as opposed to the overall mean follow-up of 4 years). Four patients have had recurrences of their decubitus ulcers. One of these was minor, requiring only a short period of local care for complete and prolonged resolution. A further surgical procedure to remove the ischial tuberosity was required in 1 case. The other 2 currently have clean and healing ulcers being treated non-operatively. All treatment decisions on these recurrent decubitus ulcers have been made in conjunction with the Department 


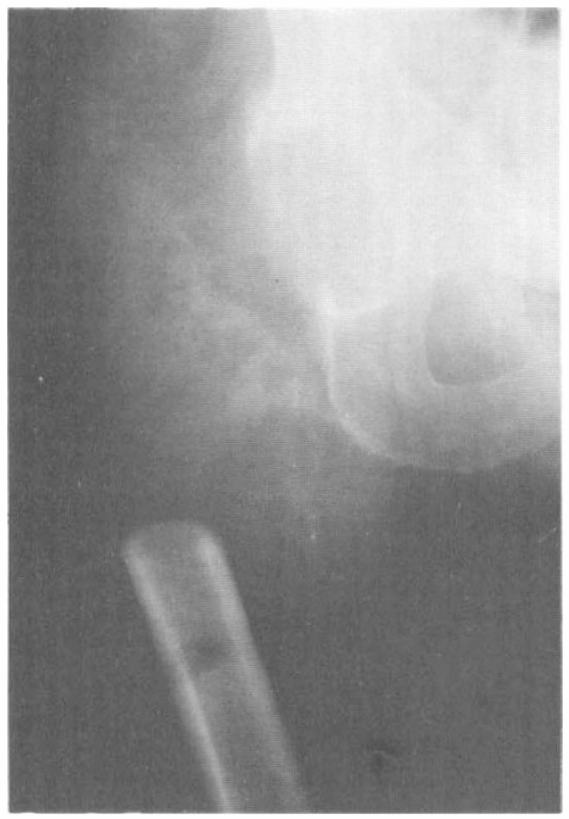

Figure 3 Post-operative radiograph illustrating level of femoral resection.

of Plastic Surgery in our hospital.

Subjective evaluation was felt to be important in this group of patients. Of the 5 patients interviewed only 1 thought that the cosmetic result of a shortened flail limb was unacceptable. Two patients felt that both sitting and transferring was improved because the movement of the hip joint was improved. All patients were able to tolerate 16 to 20 hours of wheelchair sitting and only 1 could not transfer independently, this latter patient was unable to do so preoperatively due to his C6 neurological level and was also the one who complained of the cosmetic appearance.

Objective evaluation is difficult with a small group of patients. However our data has allowed us to make an interesting observation. There was a total of 99 patient years from the time of injury to femoral resection, during which time 25 operative procedures were done for decubitus ulcers. After the femoral resection only one surgical procedure was performed in 32 patient years. Applying the Wilcoxon Rank Sum test to the patient operation data indicates a highly significant $(p<0.01)$ decrease in the amount of surgery these patients have undergone following their femoral resection. We recognise that our mean postoperative follow-up of 4 years is not equivalent to the 11 years prior to surgery but feel that it is long enough to determine developing trends. We find that this trend shows not only short term but also longer term benefit from proximal femoral resection.

\section{Conclusion}

Radical surgery is not often indicated in the treatment of spinal cord injury patients with decubitus ulcers. However, in patients with multiple failed previ- 
ous attempts at closure, insufficient tissue and underlying bony infection or hip dislocation, no other option may be suitable. Rather than hip disarticulation, limb salvage by proximal femoral resection may be recommended.

Proximal femoral resection leads to irradication of infection and healing of the decubitus ulcers. Redundant tissue becomes available for tissue transfer to deficient areas. The number of subsequent procedures necessary for decubitus ulcers is significantly reduced and half of the patients in our series have remained healed.

The patient is left with a cosmetically acceptable limb which does not interfere with wheelchair sitting or transferring, and may return to the community with fewer interruptions for hospitalisation and treatment of decubitus ulcers.

\section{References}

Baird RA, DeBenedette MJ, Eltorai I 1986 Non-septic hip instability in the chronic spinal cord injury patient. Paraplegia 24:293-300.

BENNET L, KAVNER D, LeE BY et al. 1984 Skin stress and blood flow in sitting paraplegic patients. Archives of Physical Medicine and Rehabilitation 65:186-190.

DUCHARME FA 1967 Upper femoral resection in paraplegia: indications, technique and results. Canadian fournal of Surgery 10:318-321.

EltoRAI I 1983 The Girdlestone procedure in spinal cord injured patients: A ten year experience. fournal of the American Paraplegia Society 6(4):85-86.

FERguson-Pell MW, Wilkie IC, Reswick JS et al. 1980 Pressure sore prevention for the wheelchair-bound spinal injury patient. Paraplegia 18:42-51.

FREEHAFER A 1963 Sepsis of the hip in patients with advanced neurological disease CORR29:180-188.

O'HARE JA 1964 Excision of the femoral head in the management of pressure ulcers. Proceedings of the American Clinical Spinal Cord Injuries Conference 13:97-102.

PATTERSON RP, Fisher SV 1986 Sitting pressure-time patterns in patients with quadriplegia. Archives of Physical Medicine and Rehabilitation 67:812-814.

Perkash I, O’Neill H, Politi-Meeks D et al. 1984 Development and evaluation of a universal contoural cushion. Paraplegia 22:358-365.

REICHART H 1986 Surgical treatment of pressure sores in paraplegics and possible prevention of their recurrence. Scandinavian fournal of Plastic Reconstructive Surgery 20:125-127.

RICHARDSON RR, MEYER PR JR 1981 Prevalence and incidence of pressure sores in acute spinal cord injuries. Paraplegia 19:235-247.

STEWART JC, Comarr AE 1971 Resection of head and neck of femur in spinal cord injury patients, a 25 year review. Proceedings of Veterans Administration Spinal Cord Injury Conference. 18:66-69. 